\title{
Parasitisme des petits ruminants dans la zone périurbaine de Sokodé, Togo. I. Ovins
}

\author{
P. Bastiaensen ${ }^{1 *}$ P. Dorny ${ }^{2}$ K. Batawui ${ }^{1}$ \\ A. Boukaya ${ }^{1}$ A. Napala $^{1}$ G. Hendrickx ${ }^{1}$
}

Mots-clés

Ovin - Trypanosoma - Nematoda Hématocrite - Togo.

\begin{abstract}
Résumé
Une étude parasitologique transversale menée sur 359 échantillons d'origine ovine de la zone périurbaine de Sokodé (région centrale du Togo) a permis le diagnostic de la coccidiose (prévalence de 33 p. 100) et des nématodes gastro-intestinaux (88 p. 100) représentés par Trichostrongylus sp., Cooperia sp., Oesophagostomum sp. et Haemonchus sp. En outre, des nématodes pulmonaires (Protostrongylus rufescens) ont été rencontrés (16 p. 100), ainsi que le cestode Monieza sp. (8 p. 100). Les trématodes ont été représentés par Paramphistomum sp. (15 p. 100), Fasciola sp. (1 p. 100) et Schistosoma sp. (un seul échantillon positif). Les trois agents principaux de la gale ont été présents (Sarcoptes sp., Psoroptes sp. et Chorioptes sp.). Les tiques ont été représentées par Amblyomma sp. et Boophilus sp. Des analyses sérologiques ont révélé que 22 p. 100 des animaux étaient positifs pour Toxoplasma gondii et que 79 p. 100 étaient positifs pour Oestrus ovis, malgré l'absence des larves (sondage de la cavité nasale). En combinant les effets sur l'hématocrite de différentes classes d'infections parasitaires de la trypanosomose, des nématodes gastro-intestinaux et de leurs infections mixtes, il a été constaté que la trypanosomose (13 p. 100) demeurait la parasitose ayant l'impact le plus important sur les paramètres de santé. Plusieurs analyses statistiques, qui ont pris en compte des facteurs environnementaux, génétiques et de gestion, ont permis $\mathrm{d}^{\prime}$ avoir une meilleure compréhension des interactions hôte-environnement.
\end{abstract}

1. Projet régional de lutte contre la trypanosomose animale, Direction nationale, BP 114, Sokodé, Togo.

2. Institut Prince Léopold de médecine tropicale, département de santé et de production animale, Nationalestraat 155, B-2000 Anvers, Belgique

* Auteur pour la correspondance

PO Box 145, 7270 Napier, WC, South Africa

Fax : 27 (28) 4233401 ; e-mail : office@bastiaensen.be

\section{INTRODUCTION}

La présente étude a été réalisée dans le cadre du Projet de lutte contre la trypanosomose animale au Togo (Plta). Afin de compléter les travaux extensifs menés sur la filière bovine, cette étude a eu pour but de répertorier les problèmes d'ordre parasitaire auxquels sont confrontés les éleveurs de petits ruminants qui approvisionnent la ville de Sokodé en viande de mouton ou de chèvre (5). Ainsi elle donne suite à l'étude socio-économique de l'élevage de petits ruminants dans les zones périurbaines de Lomé et de Sokodé, effectuée dans le cadre du projet Secoville en 1995, sous l'égide de la Conférence des responsables de recherche agronomique africains (Coraf). La première partie de cet article traite de la problématique chez les ovins, la deuxième partie traitera des caprins et des différences avec les ovins. 


\section{MATERIEL ET METHODES}

\section{Zone de l'étude}

La ville de Sokodé est, du point de vue démographique, la deuxième ville du Togo avec plus de 100000 habitants. La ville couvre une superficie d'environ $18 \mathrm{~km}^{2}$ avec une densité moyenne de 3372 habitants au $\mathrm{km}^{2}$ (4). La zone périurbaine de Sokodé est délimitée par l'axe de la route nationale 1 (nord-sud) et par l'axe Bassar-Tchamba (ouest-est), ainsi que par la proximité des villes de Kara et d'Atakpamé et enfin par la Réserve de Fazao-Malfakassa et la Réserve de la Forêt d'Abdoulaye, dans lesquelles ne se pratique en principe aucune activité économique. Cette zone est ainsi délimitée par un rayon d'environ $50 \mathrm{~km}$ autour de Sokodé et elle regroupe les villages de Blitta, Sotouboua, Lama-Tessi, Tchamba, Kambolé et Bassar.

\section{Elevage}

L'élevage était de type sédentaire, semi-extensif. Les infrastructures présentes sur pratiquement toutes les fermes étaient les parcs de nuit et/ou les abris. Les troupeaux encadrés bénéficiaient d'une amélioration des infrastructures (parcs de contention, râteliers et mangeoires). A peu près la moitié des animaux échantillonnés recevaient une complémentation alimentaire, le plus souvent les grains de coton. Les troupeaux encadrés bénéficiaient également d'une amélioration nutritionnelle par l'usage des grains de coton, mais aussi des légumineuses, des pois d'Angole et des déchets de maïs. Selon les réponses des propriétaires, 87 p. 100 des ovins bénéficiaient d'une mesure vétérinaire de contrôle à un moment ou un autre de leur vie, le plus souvent la vaccination contre la peste des petits ruminants, dans la moitié des cas un déparasitage interne et externe, dans 42 p. 100 des cas un suivi vétérinaire régulier. La grande majorité des ovins dans les troupeaux visités étaient indigènes, c'est-à-dire de race Naine Ouest-africaine ou race Djallonké. Les ovins exotiques (race sahélienne) (7 p. 100) se trouvaient exclusivement dans des troupeaux encadrés. La répartition des échantillonnages entre les deux sexes reflétait la composition de la plupart des troupeaux, qu'ils aient été encadrés ou villageois. Les mâles représentés étaient soit des géniteurs, soit de jeunes animaux au stade de croissance. La pyramide d'âge résumait bien les préférences des éleveurs rencontrés ; les mâles étaient destinés presque exclusivement à l'embouche à partir de l'âge d'un an. La sélection des femelles pour l'embouche ou pour la reproduction se faisait apparemment après deux ans.

\section{Animaux}

Les races ovines rencontrées ont été les suivantes : Djallonké ou race Naine de l'Afrique de l'Ouest (type local, guinéen, trypanotolérant) et Fulani ou Ouda (Uda) de type sahélien (exotique, trypanosensible) $(30,39)$. Selon les résultats du recensement agricole de 1999 (1), il y avait dans la région centrale 33968 ovins. Basé sur une extrapolation à partir du canevas régulier utilisé par le Plta, le nombre d'ovins dans la zone de l'étude avait été évalué à 19600. L'échantillonnage minimal avait été fixé à $300(29,32)$, l'objectif ayant été d'obtenir environ la moitié des données dans des troupeaux à gestion améliorée, encadrés par le Projet national de petit élevage (Pnpe), par le Plta ou par une organisation non gouvernementale ou une association d'éleveurs, et d'obtenir l'autre moitié des troupeaux villageois, constitués des ovins domestiques de plusieurs villageois. Les troupeaux du premier groupe étaient bien connus et répertoriés ; ils bénéficiaient de visites régulières d'un agent vétérinaire ou zootechnicien qui assurait le suivi technique de ces exploitations. Les troupeaux villageois n'étaient pas ou étaient très peu connus. Ces derniers ont donc été regroupés, après une première visite de sensibilisation qui devait amener les propriétaires à participer aux échantillonnages. Une première série d'échantillonnages s'est déroulée en début de saison sèche (octobre-novembre 1996), une deuxième en début de saison des pluies (avril-mai 1997). Parmi les animaux présentés, seuls les animaux sevrés étaient échantillonnés. Un nombre d'environ 20 têtes par village ou par troupeau a été échantillonné, l'objectif ayant été d'atteindre 150 mâles et 150 femelles, d'une part, 150 jeunes et 150 adultes (incisives adultes), d'autre part.

\section{Récolte de données et échantillonnage}

Un examen clinique et une anamnèse ont été effectués sur chaque animal échantillonné dans le but d'évaluer la condition générale de l'animal, de rechercher des symptômes directs ou indirects d'infestation d'ectoparasites et de récolter d'éventuels ectoparasites macroscopiques (poux, tiques) ou de réaliser des raclures dermiques (gale). La prise de sang systémique (environ $8 \mathrm{ml}$ ) a été effectuée à l'aide d'un tube hépariné sous vide (Vacutainer) dans la veine jugulaire. La prise de sang périphérique a été effectuée à l'aide d'un tube capillaire dans une veine auriculaire. Les fèces des moutons ont été récoltées par des techniciens munis d'un gant en latex ou en plastique. Les matières fécales récoltées dans les gants ont ensuite été transférées dans un bocal en plastique avec couvercle. Chaque échantillon a porté comme indication la localité, la date, l'animal, l'âge et le numéro de l'échantillon.

\section{Techniques d'analyse parasitologiques}

\section{Analyses sérologiques}

Les analyses ont concerné la recherche d'anticorps spécifiques contre la toxoplasmose (Toxoplasma gondii) et contre l'œstrose ovine (Oestrus ovis). Dans le premier cas, il s'agissait d'une analyse réalisée par le laboratoire du Plta à l'aide du kit diagnostic Toxoscreen DA (agglutination directe). Les diagnostics d'œstrose ont été réalisés par l'Ecole nationale vétérinaire de Toulouse (France) par la technique Elisa (28).

\section{Hématocrite et protozoologie}

Le principal paramètre clinique retenu a été l'hématocrite pour lequel deux systèmes de prélèvement ont été appliqués, une prise de sang dans un tube capillaire dans la veine auriculaire (sang périphérique) et une prise de sang dans un tube Vacutainer hépariné dans la veine jugulaire. La comparaison des deux techniques a permis de choisir l'hématocrite systémique (jugulaire) comme le plus fiable.

Après centrifugation des microcapillaires héparinés (sang périphérique), le tube a été coupé un millimètre au-dessus de l'interphase caillot blanc/érythrocytes (buffy coat) afin d'y inclure la couche supérieure d'érythrocytes. Le contenu de la fraction supérieure a été étalé sur une lame recouverte d'une lamelle et a été observé au microscope binoculaire (x 40) (25). Un minimum de 50 champs par lame a été examiné. Les frottis sanguins à partir de sang jugulaire ont été fixés et colorés à l'HémaColor et ensuite observés au microscope binoculaire $(10 \times 40)(7)$.

\section{Coprologie}

La coproscopie quantitative a été réalisée à l'aide de la lame de McMaster (microscope 10 x 40), le liquide de flottaison utilisé étant une solution saline dense $(d=1,20)$ qui ne concentre pas les œufs de trématodes. Ces derniers ont été recherchés à l'aide de la technique de sédimentation (22). Les nématodes gastro-intestinaux ont été différentiés par la technique de la coproculture de larves du stade L3 (35) (microscopie $10 \times$ 40, après traitement au lugol et au lactophénol ; 22). Les larves des nématodes pulmonaires ont été récoltées selon la technique de Baermann (18). Les larves ont été identifiées sous microscope suivant les clés fournies par Thienpont et coll. (37). 


\section{Entomologie}

Les tiques ont été arrachées manuellement sur les animaux et identifiées selon les clés établies par Morel (24). Les poux et les puces ont été récoltés manuellement sur les animaux et identifiés, comme pour les raclures dermiques, selon les clés établies par la Maff (22).

\section{Analyse des résultats}

Dans le cadre de cet article, seuls les résultats parasitologiques sont présentés. Ces résultats ont été analysés par rapport aux facteurs biologiques ou environnementaux recueillis pour les liaisons les plus pertinentes : hématocrite, race, sexe, âge, état physiologique (lactation, gestation, croissance), encadrement, soins vétérinaires, alimentation, saison. Les analyses statistiques (analyse à un facteur et modèle linéaire généralisé $(\mathrm{Glm})$ ont été réalisées à l'aide du logiciel Spss, version 10.0.5.

\section{RESULTATS}

Sur les 359 animaux échantillonnés, 177 provenaient de troupeaux villageois et 182 de troupeaux encadrés par le Pnpe ou un autre projet. Il s'agissait de 76 mâles et 283 femelles, 110 jeunes (moins de 14 mois) et 248 adultes.

\section{Protozoaires}

Le tableau I résume les résultats des analyses sanguines en prenant en compte la plus sensible des techniques et l'hématocrite jugulaire. La prévalence moyenne de la trypanosomose (toutes espèces confondues) a été de 13 p. 100. Deux espèces ont été identifiées : Trypanosoma congolense (9,19 p. 100) et T. vivax (5,85 p. 100). Sur 47 infections trypanosomiennes, sept étaient des infections

\section{Tableau I}

Prévalence de Trypanosoma sp. et hématocrite moyen des cas positifs

\begin{tabular}{|c|c|c|c|}
\hline Espèce & $\mathbf{N}$ & $\mathbf{n}$ & $\%$ \\
\hline T. vivax & 359 & 21 & 5,85 \\
\hline T. congolense & 359 & 33 & 9,19 \\
\hline T. brucei & 359 & 0 & 0 \\
\hline T. melophagium & 359 & 0 & 0 \\
\hline T. congolense seul & 359 & 26 & 7,24 \\
\hline T. vivax seul & 359 & 14 & 3,90 \\
\hline$T$. congolense $+T$. vivax & 359 & 7 & 1,95 \\
\hline Trypanosoma sp. & 359 & 47 & 13,09 \\
\hline $\begin{array}{l}\text { Nb. moyen de } \\
\text { trypanosomes par champ } \\
\text { microscopique } \\
\text { (pour les cas positifs) }\end{array}$ & & 4, & \\
\hline $\begin{array}{l}\text { Prévalence de la } \\
\text { trypanosomose des animaux } \\
\text { ayant un hématocrite jugulaire } \\
\text { en dessous de } 25 \%\end{array}$ & 163 & 36 & 22 \\
\hline $\begin{array}{l}\text { Hématocrite jugulaire } \\
\text { moyen des cas positifs }\end{array}$ & & 47 & $\begin{array}{r}21,04 \\
(6,09)\end{array}$ \\
\hline
\end{tabular}

$\mathrm{N}=$ nombre total d'animaux examinés

$\mathrm{n}=$ nombre d'animaux positifs mixtes à $T$. congolense et $T$. vivax. Une première indication de l'impact de la trypanosomose sur l'hématocrite moyen a été donnée par l'hématocrite moyen des cas positifs qui a été de 21 p. 100 par rapport à 24 p. 100 en moyenne. Inversement, la prévalence de la trypanosomose chez les ovins anémiés (ayant un hématocrite de moins de 25 p. 100) a été de 22 p. 100 au lieu de 13 p. 100 en moyenne. L'impact de la trypanosomose sur l'hématocrite moyen a été très significatif $(<0,005)$ chez les animaux de race Djallonké. Les infections par Trypanosoma vivax et Trypanosoma congolense seuls, ainsi que les infections mixtes ont eu un impact significatif sur l'hématocrite moyen. L'effet synergique des infections mixtes a semblé causer une baisse importante de l'hématocrite moyen (17,29 p. 100).

Théoriquement, la prévalence de la trypanosomose peut être diminuée par la pratique d'un supplément alimentaire. Ceci a été confirmé dans les troupeaux villageois (diminution de la prévalence de 18,35 à 5,88 p. 100) pour lesquels l'effet a été significatif. Dans les troupeaux encadrés, en revanche, ont été relevées des prévalences assez élevées qui étaient peu influencées ou qui ne l'étaient ni par l'amélioration de la ration alimentaire, ni par la gestion améliorée. La seule exception a concerné les troupeaux encadrés qui élevaient des ovins exotiques ou croisés, c'est-à-dire trypanosensibles, car la prévalence de la trypanosomose a été soit nulle (races sahéliennes) soit faible (croisements). Aucune autre hémoparasitose d'ordre protozoaire n'a pu être révélée par les examens des frottis sanguins.

Parmi les autres maladies protozoaires, a été relevée la présence de la coccidiose (intestinale) causée par le genre Eimeria (tableau II). Des anticorps contre Toxoplasma gondii ont été trouvés chez 22 p. 100 des ovins examinés (titre de 1/40) en saison sèche (tableau II). Les analyses sérologiques n'ont pas été réalisées en saison des pluies. Sur 13 troupeaux visités, six étaient indemnes et sept étaient infestés.

\section{Nématodes}

Des microfilaires ont été rencontrés occasionnellement sur les lames à frais ou sur les lames préparées. Leur prévalence apparente était de 1,67 p. 100 (six cas sur 359 lames examinées). Les nématodes gastro-intestinaux ont été représentés par les Strongyloides, la famille des Trichuridae, des Capillariidae, mais surtout des Trichonematidae et des Trichostrongylidae ; parmi ces deux derniers ont été trouvés, grâce à l'examen coprologique et l'identification des larves (L3), les espèces suivantes : Trichostrongylus sp., Cooperia sp., Oesophagostomum sp. et Haemonchus sp. Les espèces les plus fréquemment rencontrées ont été Haemonchus sp. et Trichostrongylus sp. (tableau III).

La saison sèche a eu un effet très négatif sur la prévalence des infestations gastro-intestinales et, dans une moindre mesure, sur les excrétions d'œufs de nématodes. Si la prévalence en saison des pluies a été de 100 p. 100, elle a diminué à 85 p. 100 en saison sèche. Les œufs par gramme de matière fécale (opg) moyens ont évolué de 3710 en saison des pluies à 2320 en saison sèche (tableau IV).

Les résultats présentés dans le tableau $\mathrm{V}$ sont les moyennes géométriques du taux d'infestation exprimées en opg. Les opg individuels ont été transformés selon la formule $\mathrm{Ln}(\mathrm{opg}+1)$. Il s'est avéré que l'influence saisonnière a été encore plus prononcée qu'en utilisant des opg moyens, mais que, en outre, les facteurs alimentation, soins vétérinaires, sexe, âge et état physiologique ont influencé de façon significative les opg transformés.

Le niveau d'excrétion d'œufs (et par conséquent le taux d'infestation par les nématodoses) $(9,23)$ a eu un effet négatif sur 
l'hématocrite moyen des animaux affectés. Cette relation n'a été significative que lorsque les différents taux ont été classés selon leur impact pathologique et spoliateur sur l'animal (niveau faible, moyen ou élevé). La figure 1 combine les effets de la trypanosomose et des nématodoses gastro-intestinales sur l'hématocrite moyen des animaux.

Les nématodes pulmonaires récoltés et identifiés lors des enquêtes en saison sèche appartenaient tous à l'espèce Protostrongylus rufescens. Aucun rapport avec des signes cliniques ou l'état de santé des animaux n'a pu être montré, malgré une prévalence de 16 p. 100 (tableau II).

\section{Cestodes}

Les cestodes ont été représentés exclusivement par le genre Monieza (prévalence de 8 p. 100).

\section{Tableau II}

Récapitulatif de la prévalence (directe ou sérologique)

des différentes espèces parasitaires (par catégorie)

\begin{tabular}{|c|c|c|c|}
\hline & $\mathbf{N}$ & $\mathbf{n}$ & Prévalence \\
\hline \multicolumn{4}{|l|}{ Protozoaires } \\
\hline Trypanosoma sp. & 359 & 47 & 13 \\
\hline Eimeria sp. & 307 & 100 & 33 \\
\hline Toxoplasma gondii & 260 & 56 & 22 \\
\hline \multicolumn{4}{|c|}{ Nématodes gastro-intestinaux } \\
\hline Strongles digestifs & 305 & 270 & 88,52 \\
\hline \multicolumn{4}{|l|}{ Nématodes pulmonaires } \\
\hline Protostrongylus rufescens & 276 & 44 & 15,94 \\
\hline \multicolumn{4}{|l|}{ Cestodes } \\
\hline Monieza sp. & 306 & 23 & 7,52 \\
\hline \multicolumn{4}{|l|}{ Trematodes } \\
\hline Fasciola sp. & 177 & 2 & 1,13 \\
\hline Paramphistomum sp. & 177 & 27 & 15,25 \\
\hline Schistosoma sp. & 177 & 1 & 0,56 \\
\hline \multicolumn{4}{|l|}{ Gale } \\
\hline Suspicion clinique & 359 & 12 & 3,34 \\
\hline Confirmation microscopique & 359 & 5 & 1,39 \\
\hline Sarcoptes sp. & 5 & 2 & \\
\hline Chorioptes sp. & 5 & 2 & \\
\hline Psoroptes sp. & 5 & 1 & \\
\hline \multicolumn{4}{|l|}{ Tiques } \\
\hline Prélèvements & 359 & 38 & 10,58 \\
\hline Amblyomma sp. & 38 & 5 & \\
\hline Boophilus sp. & 38 & 33 & \\
\hline \multicolumn{4}{|l|}{ Poux } \\
\hline Damalinia ovis & 359 & 1 & 0,28 \\
\hline \multicolumn{4}{|l|}{ OEstrose (O. ovis) } \\
\hline Suspicion clinique & 359 & 134 & 37,33 \\
\hline Détection de larves (sondage) & 134 & 0 & 0 \\
\hline Sérologie & 258 & 205 & 79,46 \\
\hline
\end{tabular}

$\mathrm{N}=$ nombre total d'animaux examinés

$\mathrm{n}=$ nombre d'animaux positifs

\section{Tableau III}

Résultats des examens coproscopiques (œufs) et des coprocultures (larves)

\begin{tabular}{lcc} 
Genre & Opg moyen & Ecart-type \\
\hline Strongyloides & 105,1 & 634,1 \\
Trichuris & 2,7 & 25,5 \\
Capillaria & 0,3 & 2,8 \\
\hline \multirow{2}{*}{ Strongles digestifs } & $\mathbf{2 5 2 4 , 2}$ & 4862,2 \\
& & \\
& Espèce (coproculture L3) & \% \\
\cline { 2 - 3 } & Cooperia sp. & 2 \\
& Haemonchus sp. & 45 \\
& Oesophagostomum sp. & 2 \\
& Trichostrongylus sp. & 51
\end{tabular}

Opg = œufs par gramme de matière fécale

Coproculture : détermination réalisée sur 1163 larves L3

\section{Tableau IV}

Impact du facteur saison sur la prévalence (\%) et le taux d'infestation (opg) des nématodes gastro-intestinaux

\begin{tabular}{lccccc} 
& N & n & Prévalence & $\begin{array}{c}\text { Opg } \\
\text { moyen }\end{array}$ & Ecart-type \\
$\begin{array}{lccccc}\text { Saison } \\
\text { des pluies }\end{array}$ & 68 & 68 & 100 & 3710,29 & 5184,27 \\
$\begin{array}{l}\text { Saison } \\
\text { sèche }\end{array}$ & 237 & 202 & 85 & 2323,73 & 4919,25 \\
\hline
\end{tabular}

$\mathrm{N}=$ nombre d'animaux examinés

$\mathrm{n}=$ nombre d'animaux positifs

Opg = œufs par gramme de matière fécale

Prévalence (Anova) : $p=0,0007 ;$ opg moyen : $p=0,044$

\section{Tableau V}

Impact des principaux facteurs recensés sur le taux d'infestation par les nématodes gastro-intestinaux (après transformation logarithmique)

\begin{tabular}{llc} 
Facteur & Variable & Opg \\
transformé ln \\
\hline \multirow{2}{*}{ Saison } & Saison des pluies & 1088 \\
& Saison sèche & 298 \\
& Appliqués & 318 \\
Sexe & Non-appliqués & 1602 \\
& Mâle & 729 \\
Etat physiolinaires & Femelle & 336 \\
& Gestation & NS \\
& Lactation & 613 \\
& Croissance & 993
\end{tabular}

Opg = œufs par gramme de matière fécale $\mathrm{NS}=$ non significatif $(\mathrm{p}>0,05)(\mathrm{Glm})$ 


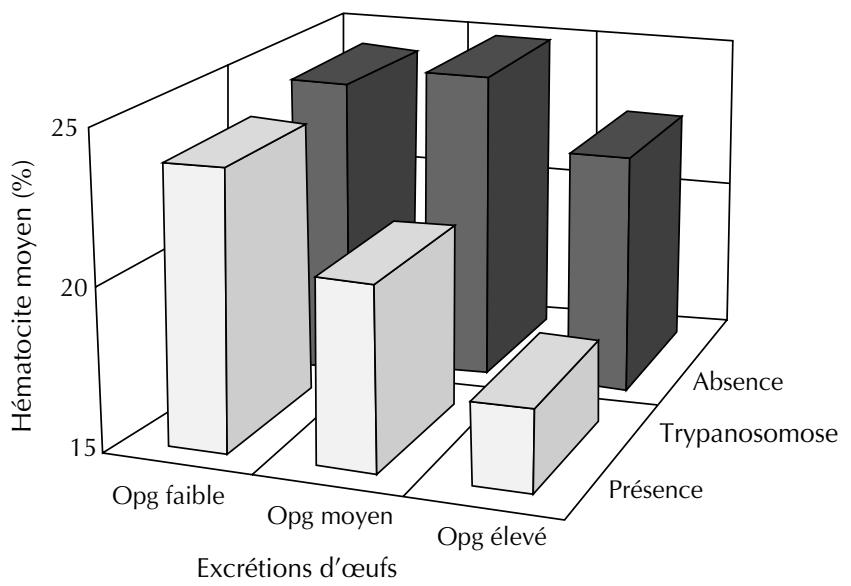

Figure 1 : hématocrite moyen (\%) pour six classes d'interférence entre la trypanosomose (en termes de présence ou $d^{\prime}$ absence) et les nématodoses gastro-intestinales (en termes $d$ 'infestations faibles, moyennes ou élevées) ; $N=285$; $p=0,002$.

\section{Trématodes}

Les trématodes ont été représentés par plusieurs genres. Celui qui a été le plus fréquemment rencontré a été Paramphistomum sp. (15,25 p. 100). Fasciola sp., la douve hépatique, n'a été rencontrée qu'à deux reprises (1,13 p. 100). Schistosoma sp. n'a été rencontrée qu'une seule fois (tableau II).

\section{Ectoparasites}

Parmi les cinq cas confirmés de gale, les trois espèces principales ont pu être identifiées : Sarcoptes sp., Chorioptes sp. et Psoroptes sp. Les deux genres de tiques rencontrés dans le cadre de cette étude sur les ovins ont été Boophilus (87 p. 100) et Amblyomma (13 p. 100). Les tiques rencontrées sur 11 p. 100 des animaux ont été récoltées essentiellement sur la peau interdigitale, ainsi que sur les oreilles. Amblyomma a été récolté essentiellement en saison des pluies, alors que Boophilus a été dominant en saison sèche (tableau II). Malgré un examen minutieux de tous les animaux échantillonnés, presque aucun animal n'a semblé être affecté ni infesté par des poux, ni par des puces. La seule espèce de poux récoltée était Damalinia ovis, une espèce qui cause une légère irritation dermique à l' animal.

Aucun cas d'œstrose ovine n'a pu être confirmé par la récolte des larves, ni par la capture de la mouche. En revanche, les analyses sérologiques réalisées à l'Ecole nationale vétérinaire de Toulouse ont révélé que 79 p. 100 des ovins présentaient des anticorps antiœstrose (205 sur 258 échantillons examinés). Le titre moyen des anticorps par rapport aux contrôles du laboratoire (100) ont été de 58,6 (écart-type de 44,0) avec un maximum de 207 (tableau II).

\section{DISCUSSION}

La prévalence moyenne de la trypanosomose a été de 13 p. 100. La prévalence moyenne chez les bovins dans la même zone périurbaine a été de 9,43 p. 100 (20). Deux espèces ont été identifiées : Trypanosoma congolense et $T$. vivax. Ces résultats sont en accord avec les résultats des enquêtes protozoologiques que le Plta avait menées dans le cadre de ses opérations de lutte dans les années 1990-1993 (19). Dans les troupeaux encadrés qui élevaient des ovins exotiques ou croisés, c'est-à-dire trypanosensibles, la prévalence de la trypanosomose a été soit nulle (races sahéliennes) ou faible (croisements). Ceci est sans aucun doute attribuable à un suivi vétérinaire intensifié, sans lequel les races exotiques ne peuvent pas se maintenir dans le milieu. Ce phénomène explique aussi l'hématocrite de 28,21 p. 100 chez les races sahéliennes.

Aucune autre hémoparasitose n'a pu être montrée par les examens des frottis sanguins, et ce, malgré la présence de vecteurs reconnus comme Boophilus et Amblyomma sp. Les seuls effets environnementaux ayant eu un impact significatif sur la prévalence des tiques ont été l'encadrement, la complémentation alimentaire et la lactation chez les brebis. Il est possible que le même mécanisme immunosuppressif qui cause une infestation élevée de nématodoses chez les brebis en lactation ait été à l'origine de cette prévalence élevée de tiques. Etant donné que l'étude n'a pu montrer la présence d'un agent parasitaire (protozoaire) transmissible par les tiques, il semble que l'impact principal des tiques ait été l'hémophagie et les infections secondaires. Toutefois, il est important de rappeler que les tiques du genre Amblyomma sont responsables de la transmission de Cowdria ruminantium ou d'Ehrlichia ruminantium, qui est une rickettsiose aiguë et souvent mortelle (cowdriose) (38). Un test sérologique en cours d'évaluation au Cirdes à BoboDioulasso (Burkina Faso) (F. Stachurksi, commun. pers.) serait en mesure de donner des indications sur la prévalence des anticorps contre $C$. ruminantium chez les ovins qui ont pu surmonter le stade clinique. De même, des tests Pcr ont été développés afin de pouvoir évaluer le taux d'infection des vecteurs $(15,31)$. Par ailleurs, des recherches sérologiques d'anticorps contre Anaplasma marginale (ou A. ovis) pourraient fournir des informations complémentaires sur l'absence apparente d'anaplasmoses dans le sang des animaux échantillonnés.

Des anticorps contre Toxoplasma gondii ont été retrouvés chez 22 p. 100 des ovins examinés en saison sèche. La présence de la toxoplasmose semble être liée aux troupeaux, plutôt qu'aux animaux individuels. Ainsi, une prévalence de 68 p. 100 a été rencontrée dans certains troupeaux. La maladie ayant surtout un intérêt en tant que zoonose, il importe d'évaluer cette prévalence chez les ovins par rapport à la prévalence chez l'homme qui varie, selon Deniau et coll., entre 62 et 88 p. 100 de séropositifs chez les femmes Togolaises entre 15 et 33 ans (10).

Les résultats de l'analyse sérologique ciblée sur Oestrus ovis justifient les soupçons des agents sur le terrain qui ont constaté un jetage nasal chez 37 p. 100 des animaux examinés. Ce même phénomène a été décrit par Ndamukong et coll. (26) pour le Cameroun où 69 p. 100 des ovins examinés ont manifesté ce jetage. Il ressort de l'étude sérologique que 79 p. 100 des ovins ont présenté des anticorps anti-œstrose (205 sur 258 échantillons examinés), ce qui est en accord avec l'enquête sérologique menée par Dorchies et coll. (11) sur des échantillons en provenance d'une dizaine de pays africains, dont le Togo pour lequel la prévalence a été de 88,6 p. 100. La différence entre la moyenne des titres Elisa (en pourcentage) pour le groupe d'animaux ayant manifesté un jetage nasal muco-purulent et celui n'ayant pas manifesté ce jetage, a été significative $(p=0,0052)$. Les auteurs n'ont pas pu montrer de différence significative entre les prévalences sérologiques des deux groupes. Aucun des facteurs recensés n'a semblé intervenir sur la prévalence sérologique de la maladie malgré certaines tendances qui se sont dégagées par rapport à l'âge et à la race.

Cette étude a confirmé la présence dominante des espèces de Haemonchus et de Trichostrongylus, présence relevée en 1995 par Bonfoh et coll. (6) pour la région des plateaux (sud du Togo) et par d'autres auteurs pour la sous-région $(2,8,12,14,17,27,34)$. La découverte d'œufs de Capillaria sp. dans les examens fécaux était 
peut-être attribuable à la consommation accidentelle de déchets avicoles, car ils n'ont jamais été diagnostiqués à ce jour. Contrairement à Bonfoh et coll. (6), la présente étude n'a pas pu montrer la présence de Gaigeria sp., qui ne peut être identifiée au travers des larves L3 (22). De façon générale, la saison sèche a eu un effet négatif sur la prévalence des infestations gastro-intestinales et sur les excrétions d'œufs de nématodes, probablement dû au phénomène d'hypobiose avec la présence de larves quiescentes (6). Il s'est avéré que l'influence saisonnière était encore plus prononcée en utilisant des moyennes géométriques $(\ln [o p g+1])$, mais qu'en outre les facteurs alimentation, soins vétérinaires, sexe, âge et état physiologique influençaient de façon significative les opg transformés. Compte tenu de la sensibilité apparente des jeunes animaux et des animaux en état de croissance pour des infestations vermineuses, il est sans doute raisonnable d'attribuer l'effet sexe à cette catégorie de jeunes animaux mâles qui constituaient la majorité des mâles au sein des troupeaux, bien qu'Asanji (2) ait déjà décrit l'effet sexe en Sierra Leone. Les excrétions élevées chez les ovins en état de lactation pourraient être attribuées au phénomène d'augmentation des opg lors du péripartum, phénomène d'immunosuppression qui affecte les brebis après le vêlage $(3,21,33)$.

Une prévalence élevée des infestations vermineuses par des nématodes gastro-intestinaux peut être améliorée par une action simultanée de gestion améliorée et de complémentation alimentaire. En ce qui concerne la présente étude, les résultats les plus encourageants ont été obtenus dans les troupeaux de races croisées et de races exotiques. L'effet sur les races indigènes a été négligeable. L'effet sur les excrétions d'œufs de nématodes a suivi la même logique : une action simultanée de gestion améliorée et de complémentation alimentaire a été bénéfique en termes d'excrétions d'œufs (et probablement d'infestations de vers adultes).

En combinant les effets de la trypanosomose et des nématodoses gastro-intestinales sur l'hématocrite moyen des animaux, il a été constaté que l'impact des nématodes était important, mais que la trypanosomose avait toutefois l'impact le plus négatif sur la santé des ovins. Les résultats ont confirmé aussi l'existence d'une synergie entre les deux parasitoses causant des effets plus dévastateurs que l'effet supposé, basé sur l'impact de chacune de ces parasitoses, ce qui a été montré par de nombreux auteurs $(13,16,36)$.

Les trématodes ont été représentés par plusieurs genres, dont principalement Paramphistomum sp. Son effet est surtout spoliateur, mais il ne représente pas de risque pathologique élevé. Comme le constataient aussi Bonfoh et coll. en 1995 (6), ni la fasciolose, ni la schistosomose ne semblent jouer un rôle important.

Malgré la reconnaissance de plusieurs cas cliniques qualifiés de gale, les résultats des analyses des raclures dermiques prises à la périphérie des plaies n'ont pu confirmer la présence de l'agent parasitaire que dans cinq cas cliniques sur douze. Il s'agissait donc soit d'une technique de dépistage trop peu sensible, soit d'un diagnostic erroné de la gale, par exemple dans des cas de dermatomycoses ou de plaies secondaires causées par des tiques. En effet, des formes larvaires de tiques ont été retrouvées dans des raclures dermiques d'animaux soupçonnés d'avoir la gale.

\section{- CONCLUSION}

Les résultats de cette étude transversale en milieu réel semblent confirmer les observations de nombreux chercheurs dans cette sous-région qui, à travers des recherches similaires de terrain ou des recherches contrôlées en station, arrivent aux mêmes conclusions : la trypanosomose animale reste pour le moment le fléau principal des petits ruminants en Afrique de l'Ouest. S'il est vrai que la prévalence des nématodes gastro-intestinaux est très élevée, il ressort de cette étude que son importance reste secondaire à celle des trypanosomes, dont les dégâts - en termes de baisse de l'hématocrite - restent prépondérants ; simultanément, cette étude montre de façon simple mais néanmoins convaincante que l'effet cumulatif des infections mixtes par des trypanosomes et par des nématodes gastro-intestinaux sur l'hématocrite est bien réel et considérable. Cet effet qui a déjà été montré dans des études en station (principalement avec des infections mixtes de T. congolense et de $H$. contortus) est dû au fait que la trypanosomose induit une immunosuppression qui facilite l'infection par d'autres agents pathologiques, principalement les nématodes digestifs ubiquitaires. Les résultats obtenus par le Plta en matière de contrôle de la trypanosomose bovine au Togo donnent de l'espoir pour la maîtrise - à travers une prise de conscience et une participation active des bénéficiaires - du problème de la trypanosomose ovine dans l'espace de quelques années. Néanmoins, tant que le prix des moutons et des chèvres sur le marché restera aussi peu incitatif pour les producteurs, on peut craindre que les éleveurs ne trouvent pas de justification pour le traitement régulier de leurs animaux. L'étude révèle aussi la situation épidémiologique de l'œstrose ovine, jusqu'à présent largement sous-estimée au Togo. L'impact réel de la maladie sur la production ovine n'est pas pour autant très clair. Dans l'immédiat et pour compléter l'image parasitaire, mais surtout sanitaire de l'élevage des petits ruminants au Togo, la situation (sérologique) de la peste des petits ruminants, de l'anaplasmose et de la cowdriose restent à clarifier. Une politique et un programme d'appui à l'élevage des petits ruminants restent plus que jamais nécessaires.

\section{Remerciements}

Les auteurs remercient chaleureusement tous les éleveurs, collaborateurs et techniciens qui ont participé à cette étude. Les auteurs souhaitent aussi remercier les docteurs K. Adoméfa, B. Faye, K. Tessio, Y. Pessinaba, A. Traoré, J. Slingenbergh et A. Sonhaye, MM. J.D. Keita, A. Rakotomahefason, K. Amedji et T. Lemou, et les professeurs P. Dorchies, V.S. Pandey, A. Verhulst et J. Vercruysse. Enfin, il convient de remercier l'ONU-FAO, qui exécuta le projet Plta, et la Belgique, principal bailleur de fonds dudit projet.

\section{BIBLIOGRAPHIE}

1. Aperçu de l'agriculture togolaise à travers le pré-recensement, 1997. Projet GCP/TOG/014-EC recensement national agricole. Lomé, Togo, direction des Enquêtes et statistiques agricoles.

2. ASANJI M.F., 1988. Haemonchosis in sheep and goats in Sierra Leone. J. Helminthol., 62: 243-249.

3. BAKER R.L., MWAMACHI D.M., AUDHO J.O., ADUDA E.O., THORPE W., 1998. Resistance of Galla and small East African goats in the sub-humid tropics to gastrointestinal nematode infections and the peri-parturient rise in faecal egg counts. Vet. Parasitol., 79: 53-64.

4. BARBIER J.-C., KLEIN B., 1995. Petit atlas urbain : Sokodé, ville multicentrée du Nord-Togo. Paris, France, Orstom.

5. BASTIAENSEN P., BATAWUI K., BOUKAYA A., DORNY P., HENDRICKX G., KADJASSI B.-T., NAPALA A., 1998. Etude transversale des contraintes d'ordre parasitaire au développement de l'élevage des petits ruminants en zone périurbaine au Togo. Etude de cas : zone périurbaine de Sokodé (région centrale). Rapport technique Projet GCP/RAF/347-BEL. Sokodé, Togo, CORAF / Anvers, Belgique, Institut de médecine tropicale. 
6. BONFOH B., ZINSSTAG J., ANKERS P., PANGUI L.J., PFISTER K. 1995. Epidémiologie des nématodes gastro-intestinaux chez les petits ruminants dans la région des plateaux au Togo. Revue Elev. Méd. vét. Pays trop., 48 : 321-326.

7. BOYT W.P., 1986. Guide pratique pour le diagnostic, le traitement et la prévention de la trypanosomiase animale africaine. Rome, Italie, FAO.

8. CHIEJINA S.N., FAKAE B.B., EZE P.I., 1989. Development and survival of free-living stages of gastrointestinal nematodes of sheep and goats on pasture in the Nigerian derived savannah. Vet. Res. Commun., 13: 103-112.

9. COADWELL W.J., WARD P.F.V., 1982. The use of faecal egg counts for estimating worm burdens in sheep infected with Haemonchus contortus. Parasitology, 85: 251-256.

10. DENIAU M., TOURTE-SCHAEFER C., AGBO K., DUPOUY-CAMET J., HEYER C., LAPIERRE J., 1991. Evaluation du risque de toxoplasmose congénitale au Togo. Bull. Soc. Pathol. exot., 84 : 664-672.

11. DORCHIES P., PREVOT F., DURANTON C., BERGEAUD J.P., AKAKPO J., PANGUI L.J., MISSOHOU A., DECONINCK P., OUATARA L., ROGER F., ACHI-YABA L., JACQUIET P.,1999. OEstrose du mouton et de la chèvre (Oestrus ovis Linné 1761) en Afrique : résultats d'une enquète sur 3204 sérums provenant de neuf pays. Revue Méd. vét., 150 : 463-466.

12. FABIYI J.P., OPEMAN D.B., HUTCHINSON G.W., 1989. Abundance and survival of infective larvae of the cattle nematodes Cooperia punctata, Haemonchus placei and Oesophagostomum radiatum from faecal parts in a wet tropical climate. Aust. Vet., 65: 229-231.

13. FAKAE B.B., CHIEJINA S.N., 1993. The prevalence of concurrent trypanosome and gastrointestinal nematode infections in West African Dwarf sheep and goats in Nsukka area of eastern Nigeria. Vet. Parasitol., 49: 313-318.

14. FAKAE B.B., 1990. The epidemiology of helminthosis in small ruminants under the traditional husbandry system in eastern Nigeria. Vet. Res. Commun., 14: 381-391.

15. FAROUGOU S., TOGUEBAYE B.S., TOUNKARA K., SY D., AKAKPO A.J., 1998. Epidémiologie de la cowdriose au Bénin. 1. Etude préliminaire sur la présence du germe chez les tiques vectrices. Revue Méd. vét., 149 : 953-958.

16. GOOSSENS B., OSAER S., KORA S., JAITNER J., NDAO M., GEERTS S., 1997. The interaction of Trypanosoma congolense and Haemonchus contortus in Djallonke sheep. Int. J. Parasitol., 27: 1579-1584.

17. GRABER M., PERROTIN G., 1983. Helminthes et helminthoses de ruminants domestiques d'Afrique tropicale. Paris, France, Editions du Point vétérinaire, $162 \mathrm{p}$

18. HANSEN J., PERRY B., 1995. Epidémiologie, diagnostic et prophylaxie des helminthiases des ruminants domestiques. Rome, Italie, FAO, $176 \mathrm{p}$

19. HENDRICKX G., NAPALA A., 1999. Le contrôle de la trypanosomose à la carte : une approche intégrée basée sur un système d'information géographique. Bruxelles, Belgique, Académie royale des Sciences d'Outre-Mer, p. 88. (Mémoires classe sciences naturelles, médicales, Sér. 8, vol. 24)

20. HENDRICKX G., 1999. Georeferenced decision support methodology towards trypanosomosis management in West Africa. Thèse Doct., faculté de Médecine vétérinaire, université de Gand, Belgique, $176 \mathrm{p}$
21. JANSEN J., 1990. Epidemiology and immunology of trichostrongylosis in cattle and sheep, a synthesis. Tijdschr. Diergeneeskd., 115: 1042-1050.

22. Manual of veterinary parasitological laboratory techniques, 1986 London, UK, Ministry of Agriculture, Fisheries and Food / HMSO, p. 37-39.

23. MCKENNA P.B., 1981. The diagnostic value and interpretation of faecal egg counts in sheep. N. Z. vet. J., 29: 129-132

24. MOREL P.C., 1976. Morphologie, biologie et rôle pathogène des tiques. Maisons-Alfort, France, Gerdat-lemvt, p. 6-13.

25. MURRAY M., MURRAY P.K., MCINTYRE W.I.M., 1977. An improved parasitological technique for the diagnosis of African trypanosomiasis. Trans. R. Soc. trop. Med. Hyg., 71: 325-326.

26. NDAMUKONG K.J.N., SEWELL M.M.H., ASANJI M.F., 1989. Disease and mortality in small ruminants in the North West province of Cameroon. Trop. Anim. Health Prod., 21: 191-196.

27. NDAO M., BELOT J., ZINSSTAG J., PFISTER K., 1995. Epidemiologie des helminthoses gastro-intestinales des petits ruminants dans la zone sylvo-pastorale au Sénégal. Vet. Res., 26 : 132-139.

28. OUATARA L., DORCHIES P., 1996. Prévalence sérologique de I'œstrose ovine au Burkina Faso : estimation par la technique Elisa. Revue Elev. Méd. vét. Pays trop., 49 : 219-221.

29. PARKER R.E., 1991. Introductory statistics for biology. Cambridge, UK, Cambridge University Press.

30. PAYNE W.J.A., 1990. An introduction to animal husbandry in the tropics. London, UK, Longman Scientific and Technical, p. 499-500.

31. PETER T.F., PERRY B.D., O'CALLAGHAN C.J., MEDLEY G.F., MLAMBO G., BARBET A.F., MAHAN S.M., 1999. Prevalence of Cowdria ruminantium infection in Amblyomma hebraeum ticks from heartwaterendemic areas of Zimbabwe. Epidemiol. Infect., 123: 309-316.

32. PUTT S.N.H., SHAW A.P.M., WOODS A.J., TYLER L., JAMES A.J., 1987. Veterinary epidemiology and economics in Africa; a manual for use in the design and appraisal of livestock health policy. ILCA Manual 3. Addis Ababa, Ethiopia, ILCA, $130 \mathrm{p}$.

33. ROMJALI E., DORNY P., BATUBARA A., PANDEY V.S., GATENBY R.M., 1997. Peri-parturient rise in faecal strongyle egg counts of different genotypes of sheep in North Sumatra, Indonesia. Vet. Parasitol., 68: 191-196.

34. SCHILLHORN VAN VEEN T.W., 1978. Haemonchosis in sheep during the dry season in the Nigerian savannah. Vet. Rec., 102: 364-365.

35. SKERMAN K.D., HILLARD J.J., 1966. A handbook for studies of helminth parasites of ruminants. Rome, Italy, UNDP/FAO, p. C.VII.1-4.

36. SPECHT E.J.K., 1982. The effect of double infections with trypanosomes and gastrointestinal nematodes on the productivity of sheep and goats in South Mozambique. Vet. Parasitol., 11: 329-345.

37. THIENPONT D., ROCHETTE F.R., VANPARIJS O.F.J., 1979 Diagnosis of verminosis by coprological examinations. Beerse, Belgium, Janssen Research Foundation, p. 48-67.

38. UILENBERG G., 1998. General review of tick-borne diseases of sheep and goats world-wide. Parasitologia, 39: 161-165.

39. WILSON T., 1992. Petits ruminants : production et ressources génétiques en Afrique tropicale. Rome, Italie, FAO, p. 101-139.

Reçu le 15.07.2002, accepté le 27.01.2004 


\section{Summary}

Bastiaensen P., Dorny P., Batawui K., Boukaya A., Napala A., Hendrickx G. Small Ruminant Parasitism in the Suburban Area of Sokode, Togo. I. Sheep

The cross-section parasitological analysis of 359 samples taken from sheep in the suburban area of Sokode (central area of Togo) allowed the diagnosis of coccidiosis (33\% prevalence) and gastrointestinal nematodes (88\%) such as Trichostrongylus sp., Cooperia sp., Oesophagostomum sp. and Haemonchus sp. Furthermore, lungworms (Protostrongylus rufescens) were found $(16 \%)$ as well as the cestode Monieza sp. $(8 \%)$.

毫 Trematodes were represented by Paramphistomum sp. (15\%), Fasciola sp. (1\%) and Schistosoma sp. (only one positive sample). All three major scabies parasites were confirmed (Sarcoptes sp., Psoroptes sp. and Chorioptes sp.). Ticks were represented by Amblyomma sp. and Boophilus sp. Serological analyses showed animals positive for Toxoplasma gondii $(22 \%)$ and for Oestrus ovis $(79 \%)$, although no larvae were found (exploration of the nasal cavity). When combining the effects on PCV of several classes of infection with trypanosomosis, gastrointestinal nematodes and their mixed infections, it appeared that trypanosomosis $(13 \%)$ remained the parasitosis with the highest impact on health parameters. Several statistical analyses that took into account environmental, management and breed-related factors allowed better understanding of host-environment interactions.

Key words: Sheep - Trypanosoma - Nematoda - Hematocrit Togo.

\section{Resumen}

Bastiaensen P., Dorny P., Batawui K., Boukaya A., Napala A., Hendrickx G. Parasitismo de los pequeños rumiantes en la zona peri-urbana de Sokodé, Togo. I. Ovinos

Un estudio parasitológico transversal, llevado a cabo sobre 359 muestras de origen ovino de la zona peri-urbana de Sokodé (región central de Togo) permitió el diagnóstico de la coccidiosis (prevalencia de 33\%) y de nemátodos gastrointestinales $(88 \%)$, representados por Trichostrongylus sp., Cooperia sp., Oesophagostomum sp. y Haemonchus sp. Por otro lado, fueron encontrados $(16 \%)$ nemátodos pulmonares (Protostrongylus rufescens), así como el céstodo Monieza sp. (8\%). Los tremátodos estuvieron representados por Paramphistomum sp. (15\%), Fasciola sp. (1\%) y Schistosoma sp. (una sola muestra positiva). Los tres principales agentes de la sarna estuvieron presentes (Sarcoptes sp., Psoroptes sp. y Chorioptes sp.). Las garrapatas estuvieron representadas por Amblyomma sp. y Boophilus sp. Los análisis serológicos revelaron que $22 \%$ de los animales eran seropositivos para Toxoplasma gondii y que $79 \%$ fueron positivos para Oestrus ovis, a pesar de la ausencia de larvas (por sondeo de la cavidad nasal). Combinando los efectos sobre el hematocrito de diferentes clases de infecciones parasitarias de la tripanosomosis, de los nematodos gastrointestinales y de las infecciones mixtas, se constató que la tripanosomosis $(13 \%)$ es la parasitosis con mayor impacto sobre los parámetros de salud. Varios análisis estadísticos, que tomaron en cuenta los factores ambientales, genéticos y de manejo, permitieron una mejor comprensión de las interacciones huésped-medio ambiente.

Palabras clave: Ovino - Trypanosoma - Nematoda - Hematocrito - Togo. 